\title{
Cavity QED with cold trapped ions
}

\author{
V. Bužek, ${ }^{1,2}$ G. Drobný, ${ }^{2}$ M. S. Kim, ${ }^{3} *$ G. Adam, ${ }^{4}$ and P. L. Knight ${ }^{1}$ \\ ${ }^{1}$ Optics Section, The Blackett Laboratory, Imperial College, London SW7 2BZ, England \\ ${ }^{2}$ Institute of Physics, Slovak Academy of Sciences, Dúbravská cesta 9, 84228 Bratislava, Slovakia \\ ${ }^{3}$ Max-Planck-Institut für Quantenoptik, Hans-Kopfermann-Strasse 1, 85748, Garching, Germany \\ ${ }^{4}$ Institut für Theoretische Physik, Technische Universität Wien, Wiedner Hauptstrasse 8-10, A-1040 Vienna, Austria
}

(Received 10 February 1997)

\begin{abstract}
We analyze the quantum motion of a cold, trapped two-level ion interacting with a quantized light field in a single-mode cavity. We show that in the nonclassical Lamb-Dicke limit the time evolution of the vibrational mode representing the quantized motion of the center of mass of the trapped ion is very sensitive to the quantum statistics of the light field. We also show that the system under consideration may evolve into the maximally entangled three-particle Greenberger-Horne-Zeilinger state. We briefly discuss the dynamics of a cluster of two-level ions trapped in a cavity and interacting with a quantized light field.
\end{abstract}

[S1050-2947(97)07209-0]

PACS number(s): 42.50.Ct, 32.80.Pj, 42.50.Dv

\section{INTRODUCTION}

Cavity QED as currently investigated (see, e.g., [1] and references therein) has been plagued by fluctuations in the number of quantum sources (atoms) interacting with a cavity field at any instant. These fluctuations in the number of atoms partially smear out the quantum nature of the atom-field interaction in a cavity. For example, the ac Stark effect could result in absorption spectra being split into doublets or not, depending on whether or not a single atom is present in the cavity $[2,3]$. A thermal beam of source atoms traversing the cavity will result in an essentially Poisson distribution of source atoms at any time. For this reason a number of experimental groups [4] have begun to turn their attention to the problem of a single trapped ion interacting with a single quantized cavity mode. It is now routinely possible experimentally to trap a single ion [5], and if this could be done in a high finesse optical cavity it would allow us to study cavity QED dynamics when just two precisely specified quantum systems, i.e., the trapped ion and the single-mode cavity field, are strongly coupled together. This means that within the lifetime of a photon in the cavity, this photon can be "exchanged" many times between the ion and the field. As a consequence of this interaction the two subsystems (i.e., the internal state of the trapped ion and the cavity mode) become quantum-mechanically entangled [2]. On the other hand, it has been shown recently $[6,7]$ that ions in trapping potentials interacting with classical light fields can be cooled down to their lowest vibrational states and that from these, arbitrary quantum vibrational states of trapped ions can be prepared in a controlled way $[8,9]$. Therefore, it is reasonable to assume that the single trapped ion interacting with a quantized cavity field may be cooled down to its lowest vibrational state. This would represent the "ultimate" quantummechanical system in which three quantum subsystems (i.e., the internal ionic states, the quantum vibrational mode of the

\footnotetext{
*On leave from Department of Physics, Sogang University, C.P.O. Box 1142, Seoul, Korea.
}

ion, and the single-mode cavity field) are coupled together. In the present paper we propose a simple, exactly solvable model describing this physical situation [10]. As we will see, it adds new phenomena to the subject of the cavity QED, and in particular allows for the construction of the fully correlated Greenberger-Horne-Zeilinger (GHZ) state [11] in a remarkably simple way.

A semiclassical model describing the dynamics of trapped ions interacting with a traveling-wave light field was introduced by Blockley et al. [12]. An analogous model employing standing-wave light fields was proposed by Cirac et al. [13]. In these models a single two-level ion undergoes quantized vibrational motion within a harmonic trapping potential and interacts with a classical single-mode light field. Blockley and co-workers [12] pointed out that in the Lamb-Dicke regime the dynamics of trapped ion can be described by a very simple Hamiltonian similar to that of the JaynesCummings model (JCM) [14]. Later it was shown that outside of the Lamb-Dicke regime the vibrational motion of a trapped ion can be described by a strongly nonlinear JCM [15]. Zeng and Lin have investigated the generation of nonclassical vibrational states of atomic motion in a quantized trap, based on the transfer of nonclassical features from the quantized electromagnetic field to the atomic motion [16].

Within the framework of these Jaynes-Cummings-like models, various aspects of the dynamics of trapped ions have been studied. For example, quantum nondemolition measurement of vibrational quanta of trapped ions has been analyzed theoretically [17] and several schemes proposed [18] for the reconstruction of quantum-mechanical vibrational states of a trapped ion. One of these schemes has been successfully applied to the experimental reconstruction of the Wigner function of nonclassical states of the vibrational mode of a trapped ion [19].

As noted above, there exists a very close formal analogy between an ion vibrating in a trapping potential and an ion interacting with a quantized cavity field. Therefore many ideas and effects that have been discussed within the framework of cavity QED can now be "mapped" onto the trapped-ion models and vice versa. For example, cavity-QED 
models of quantum computing [20,21], quantum state engineering [22], and quantum state endoscopy [23] should be mentioned.

In the present paper we go beyond the formal analogy between the trapped-ion and cavity-QED models. We combine them so that we can describe the interaction of a trapped ion (with a quantized motional state) with a quantized cavity light field. Our motivation for this generalization is as follows: in the process of preparation of nonclassical vibrational states of a trapped ion, an appropriate sequence of laser pulses tuned to either the ionic electronic transition or to resolved vibrational sidebands (i.e., a Raman transition between internal ionic states) are used [8]. Here the driving laser pulse is a classical field so the vibrational mode can be mutually entangled (correlated) with only the internal degrees of freedom. On the other hand, for quantum information processing, an entanglement with an additional system is often required. The channel for information exchange between ions stored in a linear trap ("ionic crystals") is given by their collective vibration mode [24]. Another possibility may be to couple ion traps (with single ions) via their common resonator (cavity), which supports one particular mode of the electromagnetic field. The cavity field mode could then be considered as the quantum channel for information transfer between ions.

The model presented in the paper provides us with a full quantum-mechanical picture of dynamics of trapped ion interacting with a single-mode electromagnetic field. In particular, the model reveals many interesting features of the quantum-mechanical entanglement between the ionic internal degree of freedom, the vibrational mode, and the light field. For example, such a configuration with three entangled subsystems enables us to create the GHZ states of central interest in quantum-measurement theory and to test quantum mechanics versus local realism (hidden variables) theories [11].

This paper is organized as follows: Section II is devoted to a brief description of the Blockley-Walls-Risken model. The model of the completely quantized system is described in Sec. III. In this section we also present the exact analytical solution of our model. In Sec. IV we consider two two-level trapped ions interacting with a single cavity mode. In Sec. V we present conclusions.

\section{TRAPPED ION INTERACTING WITH A CLASSICAL LIGHT FIELD}

First we briefly review the model proposed by Blockley et al. [12] in which a two-level ion moves in a harmonic potential and simultaneously interacts with the single-mode classical field. The corresponding Hamiltonian $\hat{H}_{B W R}$ in the frame rotating at the light field frequency reads

$$
\begin{aligned}
\hat{H}_{B W R}= & \hbar \nu\left(\hat{b}^{\dagger} \hat{b}+\frac{1}{2}\right)+\frac{1}{2} \hbar \Delta \hat{\sigma}_{z} \\
& +\frac{1}{2} \hbar \Omega\left[\hat{D}_{b}(i \epsilon) \hat{\sigma}_{+}+\hat{D}_{b}^{\dagger}(\mathrm{i} \epsilon) \hat{\sigma}_{-}\right],
\end{aligned}
$$

where $\hat{D}_{b}(\xi)=\exp \left[\hat{b}^{\dagger}-\xi^{*} \hat{b}\right]$ is the displacement operator; $\hbar \nu$ is the energy of the trap (vibrational) quanta; $\hat{b}\left(\hat{b}^{\dagger}\right)$ is
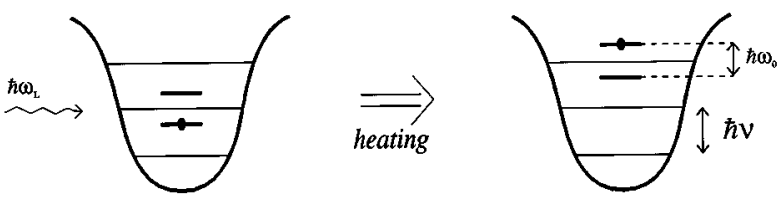

FIG. 1. A schematic description of a heating of the trapped two-level ion with the internal transition energy $\hbar \omega_{0}$ by the external light field (laser) with frequency $\omega_{L}$. The vibrational mode is excited by one quantum $\hbar \nu$. In the opposite process the ion cools down.

the annihilation (creation) operator of the quantized vibrational motion of the ion, $\hat{\sigma}_{\mp}$ and $\hat{\sigma}_{z}$ are the Pauli spin operators of the two-level ion; $\Delta=\omega_{0}-\omega_{L}$ is the detuning of the internal ionic transition at frequency $\omega_{0}$ from the laser frequency $\omega_{L} ; \Omega$ is the Rabi frequency of the driven transition in the external classical (laser) field; the parameter $\epsilon$ is defined as $\epsilon=\sqrt{E_{r} /(\hbar \nu)}$, where $E_{r}$ is the recoil energy of the ion. It is supposed that the ion during its time evolution does not leave the trap. Neither spontaneous emission nor the influence of ionic micromotion [25] is taken into account here.

This model, in the nonclassical Lamb-Dicke regime of small $\epsilon$, with the external driving field tuned to the first (upper) vibrational sideband (i.e., $\Delta=-\nu$ ) shares features similar to the JCM [14]. The difference between the BlockleyWalls-Risken [12] model and the JCM is the nature of the bosonic field to which the atom is coupled: the quantized single-mode electromagnetic field in the JCM is replaced by the quantized vibrational mode, which represents the motion of the center of mass of the ion under consideration.

We assume that the driving laser frequency is tuned to the first (blue) vibrational sideband, i.e., $\Delta=-\nu$. If the ion absorbs energy from the classical light field, this absorption process is accompanied by an excitation of the vibrational mode, which causes heating of the ion (a schematic description of the heating process of the trapped ion is depicted in Fig. 1). In the opposite process the ion emits its excitation energy into the light field and cools down. This process is accompanied by a decrease of the number of vibrational quanta. It has been shown $[12,13,26]$ that the effective interaction Hamiltonian corresponding to the Lamb-Dicke regime with transitions that involve an exchange of only one trap quantum (i.e., $\epsilon^{2} \bar{n}_{b} \ll 1 ; \bar{n}_{b}$ being the average number of the trap quanta) in the rotating-wave approximation for this detuning (i.e., $\Omega \ll \nu$ ) can be written as

$$
\hat{H}_{\text {c.f. }}=\frac{i}{2} \hbar \Omega \epsilon\left[\hat{b}^{\dagger} \hat{\sigma}_{+}-\hat{b} \hat{\sigma}_{-}\right]
$$

By analogy with the JCM $[14,27,28]$, collapses and revivals of the ionic inversion have been predicted and observed [8] when the ion, initially in a lower internal energy state, is prepared in a coherent vibrational state. In this case the revival time $t_{R}$ of Rabi oscillations is estimated as $t_{R} \approx 4 \pi \beta /(\Omega \epsilon)$, where $\beta$ is the initial amplitude of the coherent vibrational state $|\beta\rangle_{b}=\hat{D}_{b}(\beta)|0\rangle_{b}$, where $|0\rangle_{b}$ denotes the vacuum state of the vibrational mode. The behavior of the phase-space Husimi $Q_{b}$ function of the vibrational mode [see Eq. (3.3)] also exhibits very interesting features. 
Namely, it bifurcates, and at one-half of the revival time a pure superposition vibrational state is produced, i.e., the ion can be found simultaneously in two macroscopically separated states within the trap (the so-called Schrödinger cat state [29]).

One quite natural question arises: "What will happen if the single-mode light field is treated quantum mechanically?." To answer this question we propose in the present paper a fully quantum-mechanical model in which the trapped two-level ion interacts with a quantized cavity mode of the light field in a high-finesse resonator. This model takes into consideration the effects of the back action of the ion on the field mode and correlations between the ionic degrees of freedom (internal and vibrational) and the quantized cavity field.

\section{MODEL WITH QUANTIZED CAVITY FIELD MODE}

In what follows we consider the situation when the trapped two-level ion is placed in a resonator (cavity) that supports one particular mode of the electromagnetic field. The cavity field is treated quantum mechanically. In the Lamb-Dicke regime of small $\epsilon$, when the quantized field mode with frequency $\omega_{L}$ is tuned to the first (blue) vibrational sideband $(\Delta=-\nu)$, we can write the interaction Hamiltonian as

$$
\hat{H}_{\mathrm{q} . \mathrm{f} .}=\frac{i}{2} \hbar \kappa \epsilon\left[\hat{b}^{\dagger} \hat{c} \hat{\sigma}_{+}-\hat{b} \hat{c}^{\dagger} \hat{\sigma}_{-}\right]
$$

where $\hat{c}\left(\hat{c}^{\dagger}\right)$ is the annihilation (creation) operator of the quantized cavity field mode and $\kappa$ is the ion-field coupling constant in the dipole approximation. The interaction Hamiltonian (3.1) describes the process of heating, in which the absorption of one photon excites the ion and increases its vibrational energy by one quantum [the first term of the Hamiltonian (3.1) is schematically depicted in Fig. 1]. Cooling is the opposite process [described by the second term in Eq. (3.1)] in which emission of one photon deexcites the ion and decreases the number of vibrational quanta by one. We note that our model described by Eq. (3.1) involves a multiplicative trilinear Hamiltonian in which the internal ionic degrees of freedom are coupled to both the single-mode cavity field and the vibrational degrees of freedom. This should be contrasted with the two-mode vibrational coupling considered recently by Gou et al. [30], which is additive in the sense that the internal ionic degrees of freedom are coupled to one or the other bosonic mode additively. The analogous additive type of interaction has been considered by Zeng and Lin [16]. These authors have studied the far off-resonant case when internal atomic degrees of freedom can be adiabatically eliminated. In this case the effective Hamiltonian $\hat{H}_{\mathrm{eff}} \simeq i \lambda\left(\hat{b}^{\dagger} \hat{c}-\hat{b} \hat{c}^{\dagger}\right)$ describes an effective linear coupling between the two bosonic modes (i.e., the vibrational mode and the single mode of the cavity field). Obviously, this bilinear Hamiltonian differs from the trilinear Hamiltonian (3.1) discussed in the present paper. We also note that Gerry and Eberly [31] studied a trilinear Hamiltonian analogous to Eq. (3) within the context of two-photon transitions of a two-level atom interacting with a bichromatic field in a cavity.
In the present paper we compare two models when the cavity field mode is treated (1) classically [see Eq. (2.2)] and (2) quantum mechanically [see Eq. (3.1)], respectively. We will study how the initial photon statistics of the quantized cavity field mode affects the time evolution of the system under consideration as well as back action of the ion on the cavity field.

To distinguish between pure states and statistical mixtures into which the quantum-mechanical subsystems (i.e., the ionic internal and vibrational degrees of freedom, and the bosonic cavity mode) evolve, we study the time evolution of the entanglement parameter. For a particular subsystem described by the reduced density operator $\hat{\rho}_{x}=\operatorname{Tr}_{\{y \neq x\}} \hat{\rho}$ ( $\hat{\rho}$ is the density operator of the whole system) the entanglement parameter is associated with the linearized entropy [32]

$$
S_{x}^{\mathrm{corr}}=1-\operatorname{Tr}_{x}\left\{\hat{\rho}_{x}^{2}\right\} .
$$

This entropy is equal to zero for pure states and for any statistical mixture state $S_{x}^{\text {corr }}>0$. In addition, $S_{x}^{\text {corr }}$ represents a lower bound of the corresponding von Neumann entropy $S_{x}=-\operatorname{Tr}_{x}\left[\hat{\rho}_{x} \ln \rho_{x}\right]$, i.e., $S_{x}^{\text {corr }}(t) \leqslant S_{x}(t)$. Thus the entanglement parameter can be used to quantify the degree of correlation established during the interaction between quantummechanical subsystems involved in dynamics. To "visualize" quantum-statistical properties of a particular bosonic mode the phase-space Husimi $Q_{x}$ function is used. This phase-space probability density distribution is defined as [33]

$$
Q_{x}(\alpha)=\left\langle\alpha\left|\hat{\rho}_{x}\right| \alpha\right\rangle,
$$

where $|\alpha\rangle$ is a coherent state with the complex amplitude $\alpha=x+$ i $y$.

\section{A. Solution of the model}

Utilizing the existence of two integrals of motion

$$
\hat{R}_{1}=\hat{b}^{\dagger} \hat{b}+\hat{c}^{\dagger} \hat{c} \quad \text { and } \quad \hat{R}_{2}=\hat{b}^{\dagger} \hat{b}-\hat{\sigma}_{+} \hat{\sigma}_{-}
$$

associated with the Hamiltonian (3.1) (i.e., $\left[\hat{H}_{\text {q.f. }}, \hat{R}_{k}\right]=0$ )we obtain the general solution for the state vector $|\psi(t)\rangle$ of the system governed by this Hamiltonian. If we consider the initial state of the ion-field system to be described by the state vector

$$
|\psi(0)\rangle=\sum_{n} c_{n}|n\rangle_{c} \otimes \sum_{m} b_{m}|m\rangle_{b} \otimes|g\rangle_{i}
$$

then in the resonant case the general solution in the interaction picture reads

$$
\begin{aligned}
|\psi(t)\rangle= & \sum_{m, n} b_{m} c_{n}\left[\cos \left(\Omega_{m, n} t\right)|m\rangle_{b}|n\rangle_{c}|g\rangle_{i}\right. \\
& \left.-i \sin \left(\Omega_{m, n} t\right)|m+1\rangle_{b}|n-1\rangle_{c}|e\rangle_{i}\right],
\end{aligned}
$$

where $|n\rangle_{c}$ and $|m\rangle_{b}$ are number (Fock) states of the cavity field and the vibrational mode, respectively; $|g\rangle_{i}\left(|e\rangle_{i}\right)$ denotes the internal lower (upper) ionic level. The generalized Rabi frequency $\Omega_{m, n}$ is given by the relation 


$$
\Omega_{m, n}=\frac{1}{2} \kappa \epsilon \sqrt{n(m+1)} .
$$

It is instructive to start our analysis of the dynamics of the system governed by the Hamiltonian (3.1) for the initial state vector

$$
|\psi(0)\rangle=|\beta\rangle_{b} \otimes|N\rangle_{c} \otimes|g\rangle_{i},
$$

i.e., where the cavity mode is prepared in the Fock state $|N\rangle_{c}$, the vibrational mode is initially in a coherent state with amplitude $\beta$ (for simplicity we assume $\beta$ to be real) and the ion is in its internal lower state $|g\rangle_{i}$. We remind ourselves that the initial average number of trap (vibrational) quanta $\bar{n}_{b}=\beta^{2}$ has to be chosen such that the condition $\epsilon^{2} \bar{n}_{b} \ll 1$ is fulfilled. This condition implies restriction on the range of parameters for which the Hamiltonians given by Eqs. (2.2) and (3.1) are relevant. The initial state (3.8) evolves according to Eq. (3.6) with $b_{m}=e^{-\beta^{2} / 2} \beta^{m} / \sqrt{m !}$ and $c_{n}=\delta_{N, n}$. In this case the dynamics is characterized by a perfect correlation between the internal ionic energy states and the cavity field. Because of this perfect correlation, each of the two quantum-mechanical subsystems is, for any time $t>0$, in a statistical mixture. We note that $\| g(N)\rangle\rangle \equiv|N\rangle_{c}|g\rangle_{i}$ and $\left.\left.\| e(N)\right\rangle\right\rangle \equiv|N-1\rangle_{c}|e\rangle_{i}$ form collective ion-field states. The expression for the state vector $|\psi(t)\rangle$ fulfilling the initial condition (3.8) can be written as

$$
\begin{aligned}
|\psi(t)\rangle= & \left.\sum_{m} b_{m}\left[\cos \left(\Omega_{m, N} t\right)|m\rangle_{b}|| g(N)\right\rangle\right\rangle \\
& \left.\left.\left.-i \sin \left(\Omega_{m, N} t\right)|m+1\rangle_{b}|| e(N)\right\rangle\right\rangle\right] .
\end{aligned}
$$

From here it follows that the time evolution of the vibrational mode is the same as for the case of the Hamiltonian (2.2) with a classically treated field mode, providing that the parameter $\Omega$ in Eq. (2) is chosen such that $\Omega=\kappa \sqrt{N}$. This is in correspondence with an intuitive picture in which $\sqrt{N}$ is associated with the amplitude of the equivalent classical field, with no fluctuations in the intensity.

Obviously, the mean number of the trap quanta $\bar{n}_{b}(t)$ exhibits collapses and revivals analogous to the collapserevival effect in the JCM $[14,27]$. We plot $\bar{n}_{b}(t)$ as a function of the scaled time in Fig. 2(a). The corresponding revival time $t_{R}^{(b)}$,

$$
t_{R}^{(b)}(N) \approx \frac{4 \pi \beta}{\kappa \epsilon \sqrt{N}}
$$

is exactly the same as in the model with a classical field mode, governed by the Hamiltonian (2), providing $\Omega=\kappa \sqrt{N}$. In Fig. 2(b) we plot the time evolution of the entanglement parameter $S_{b}^{\text {corr }}$, which at one-half of the revival is almost equal to zero. It can be checked by direct calculations that at one-half of the revival time the vibrational mode is in a pure superposition state [12] composed of two coherentlike states that are mutually rotated around the origin of phase space by $\pi$ (the so-called Schrödinger cat state [29]).

The dynamics of the vibrational mode within the model described by the Hamiltonian (3.1) for the initial state (3.8)
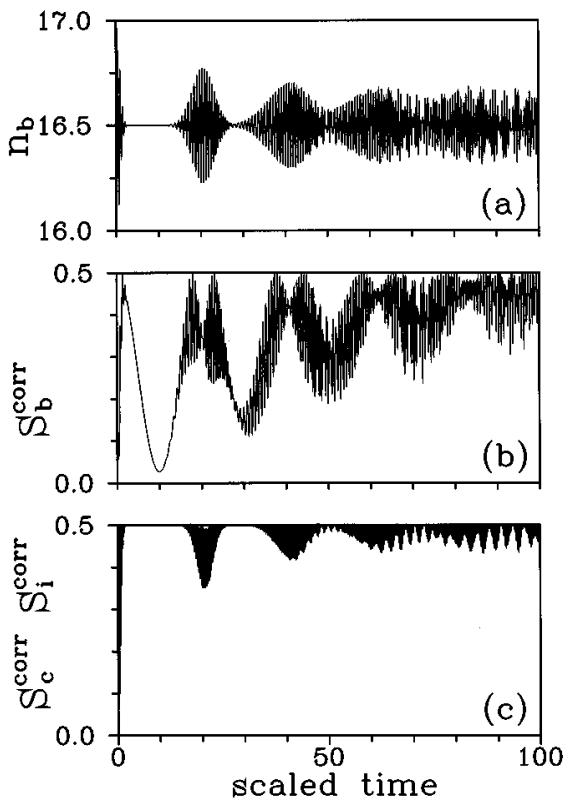

FIG. 2. (a) The time evolution of the average number of vibrational quanta $n_{b}$ and (b) the entanglement parameters $S_{b}^{\text {corr }}$ of the vibrational mode; (c) $S_{c}^{\text {corr }}$ of the cavity mode, which evolves identically with $S_{i}^{\text {corr }}$ of the internal ionic system. The initial state vector is of the form (3.8) with the cavity field in the number state $|N=64\rangle_{c}$, the vibrational mode in the coherent state $|\beta=4\rangle_{b}$, and the ion in its ground state $|g\rangle_{i}$. The scaled time is $\kappa t / 2 \pi$ and $\epsilon=0.05$.

is the same as within the model (2.2) with a classically treated light field. On the other hand, the time evolution of the internal ionic energy states is significantly affected by the quantum nature of the cavity mode. Namely, within the model (2.2) with a classical light field, the ion is, at one-half of the revival time, in the pure superposition $|\psi\rangle_{i}=(1 / \sqrt{2})$ $\times\left(|g\rangle_{i}+e^{i \varphi}|e\rangle_{i}\right)$. On the contrary, if the light field is quantized [i.e., the ion-cavity-field dynamics is governed by the Hamiltonian (3.1)] then at one-half of the revival time the ion evolves into a statistical mixture described by the density operator $\hat{\rho}_{i}=\frac{1}{2}\left(|g\rangle_{i}{ }_{i}\langle g|+| e\rangle_{i}{ }_{i}\langle e|\right)$. The corresponding entanglement parameter $S_{i}^{\text {corr }}$ is equal to $1 / 2$, which reflects the degree of mutual entanglement between the cavity mode and the internal ionic levels. This is illustrated in Fig. 2(c). Note that for the cavity mode initially prepared in a Fock state the correlation parameters $S_{c}^{\text {corr }}$ and $S_{i}^{\text {corr }}$ evolve identically. What one finds at one-half of the revival time is the Bell-like state, with perfect correlations between the internal states of the ion and the states of the cavity mode, i.e., $|\psi\rangle_{c+i}=(1 / \sqrt{2})\left(|N\rangle_{c}|g\rangle_{i}+e^{i \varphi}|N-1\rangle_{c}|e\rangle_{i}\right)$.

In our second example we will consider the cavity field to be initially prepared in a coherent state $|\gamma\rangle_{c}$. Comparing this example with the previous case when the field was supposed to be prepared in a Fock state we find that the quantum statistical properties of the cavity mode significantly affect the vibrational motion of the trapped ion. To be specific, let us consider the initial state vector

$$
|\psi(0)\rangle=|\beta\rangle_{b} \otimes|\gamma\rangle_{c} \otimes|g\rangle_{i},
$$


i.e., the vibrational and the radiation modes are prepared in coherent states with amplitudes $\beta, \gamma$ (taken to be real for simplicity) and the ion is in its lower internal state $|g\rangle_{i}$. In our discussion we will consider the initial mean number of vibrational quanta to be fixed $\left(\bar{n}_{b}=\beta^{2}\right)$ and we will assume various intensities of the initial coherent state of the cavity mode. The time evolution of the initial state (3.11) is given by Eq. (3.6) with $c_{n}=e^{-\gamma^{2} / 2} \gamma^{n} / \sqrt{n !}$ while $b_{m}$ $=e^{-\beta^{2} / 2} \beta^{m} / \sqrt{m !}$. The average number of trap quanta at time $t$ can now be written in the form

$$
\begin{aligned}
\bar{n}_{b}(t) & =\bar{n}_{b}+\sum_{n} c_{n}^{2} \overbrace{\sum_{m} b_{m}^{2} \sin ^{2}\left(\Omega_{m, n} t\right)}^{t_{R}^{(b)}} \\
& =\bar{n}_{b}+\sum_{m} b_{m}^{2} \overbrace{\sum_{n} c_{n}^{2} \sin ^{2}\left(\Omega_{m, n} t\right)}^{t_{c}^{(c)}} .
\end{aligned}
$$

Eq. (3.12) describes the double coherent summation of terms oscillating at generalized Rabi frequencies $\Omega_{m, n}$ [see Eq. (3.7)]. This Poissonian averaging results in the appearance of two time scales on which collapses and revivals of the average number of vibrational quanta appear. These two time scales are indicated in Eq. (3.12). One of them is associated with the characteristic time

$$
t_{R}^{(b)} \approx \frac{4 \pi \beta}{\kappa \epsilon \gamma},
$$

while the other time scale is given by the relation

$$
t_{R}^{(c)} \approx \frac{4 \pi \gamma}{\kappa \epsilon \beta}
$$

For $\gamma^{2} \gg \beta^{2}$, the time $t_{R}^{(c)}$ is related to revivals of the envelope of the "rapid revivals" governed by the revival time $t_{R}^{(b)}$, i.e., the rapid revivals of the mean number of vibrational quanta are modulated on the "overall" time scale associated with $t_{R}^{(c)}$. We illustrate this modulation of rapid revivals in Fig. 3(a) in which we plot the time evolution of the mean number of vibrational quanta.

The behavior of the mean vibrational quanta, and also the quantum entanglement between the ionic internal states, the ionic vibrational states, and the quantized cavity mode induced by the interaction Hamiltonian (3.1) all very sensitively depend on the quantum statistics of the light field. We have seen that when the cavity mode is initially prepared in the Fock state, then at $t=t_{R}^{(b)} / 2$ the linearized entropy of the vibrational mode $S_{b}^{\text {corr }}$ is approximately equal to zero, which means that the vibrational mode during its time evolution evolves into a pure state. On the contrary, as can be seen from Fig. 3(b), if the cavity mode is initially prepared in a coherent state, then the entanglement parameter of the vibrational mode is significantly larger than zero for any $t>0$, i.e., the vibrational mode is (in this case) always in a statistical mixture. We note that if $\gamma, \beta \gg 1$ then the $Q_{b}$ function of the vibrational mode bifurcates into two components. Moreover, as seen from Fig. 4(a), at one-half of the characteristic time $t_{R}^{(b)}$, this probability density distribution has two "macro-
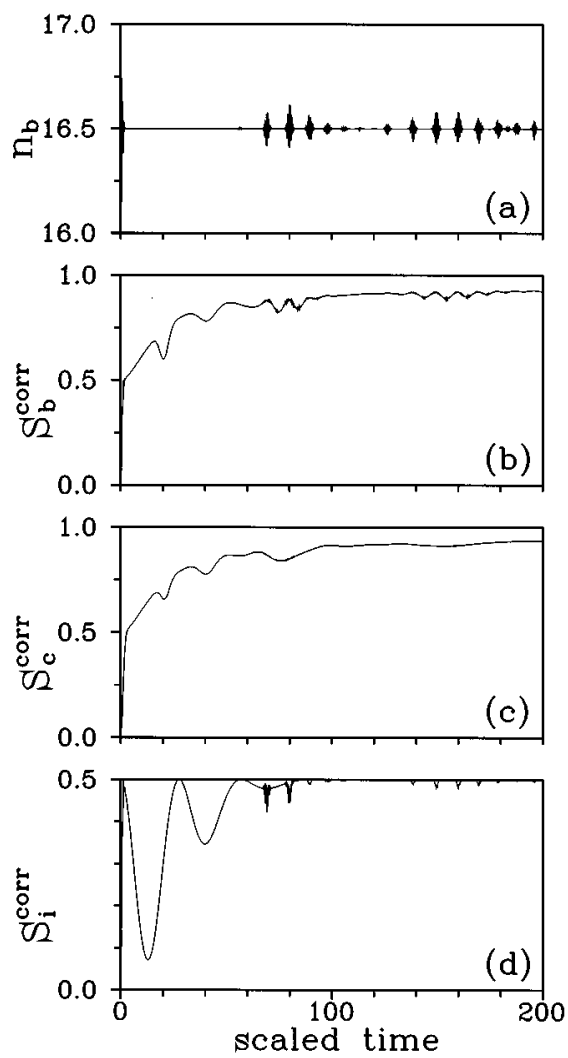

FIG. 3. The cavity mode prepared in the coherent state $|\gamma=8\rangle_{c}$. (a) time evolution of $n_{b}$; (b) $S_{b}^{\text {corr }}$; (c) $S_{c}^{\text {corr }}$; (d) (c) $S_{i}^{\text {corr }}$. Other parameters are the same as in Fig. 2.

scopically" distinct peaks in phase space. If we set $\Omega=\kappa \gamma$ [this substitution corresponds to the naive semiclassical replacement of $\hat{a} \rightarrow \gamma$ in the Hamiltonian (3.1)] then the time scale associated with the characteristic time $t_{R}^{(b)}$ is equal to the revival time in the model (2.2) when the light field is treated classically. Setting the two time scales equal we see that at $t_{R}^{(b)} / 2$ the $Q_{b}$ functions in both models have two distinct peaks. We stress that the $Q_{b}$ function of the vibrational mode in the fully quantized model describes a statistical mixture, while in the Blockley-Walls-Risken model the corresponding $Q_{b}$ function describes a pure superposition state. For any $\gamma \neq 0$ the process governed by the Hamiltonian (3.1) is characterized by an inevitable loss in the initial purity of the states of the bosonic subsystems, due to their mutual
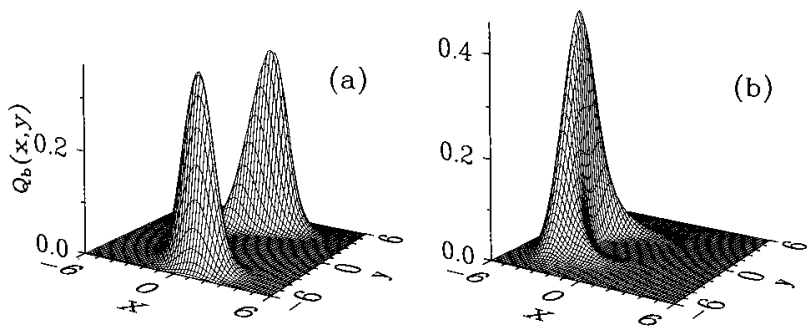

FIG. 4. The $Q_{b}$ function of the vibrational mode (a) at the half of the revival time and (b) at the revival time $t_{R}^{(b)}$. The cavity field is prepared initially in the coherent state $|\gamma=8\rangle_{c}$. Other conditions are the same as in Fig. 2. 

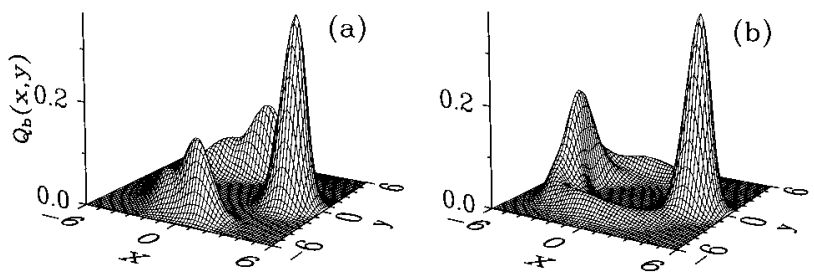

FIG. 5. The $Q_{b}$ function of the vibrational mode (a) at the half of the revival time and (b) at the revival time $t_{R}^{(b)}$. The cavity field is prepared initially in the coherent state $|\gamma=1\rangle_{c}$. Other conditions are the same as in Fig. 2.

entanglement. The only exception is the internal ionic degree of freedom. As Fig. 3(d) suggests for $\gamma^{2} \gg \beta^{2} \gg 1$ an almost pure ionic coherent state is produced at $\frac{1}{2} t_{R}^{(b)}$. Note that simultaneously a pure state in the "complementary" system composed of the vibrational and cavity modes has to appear.

For example, with $\gamma=2 \beta$ one finds at $t_{R}^{(b)} / 2$ a structure close to a pure two-mode Schrödinger catlike state for the vibrational and cavity modes, which consists of four components. Each particular bosonic mode is in a two-component mixture (in the vibrational mode the components are mutually rotated by $\pi$ at $t_{R}^{(b)} / 2$ while in the cavity field mode they are rotated by $\pi / 4$ as $\left.\frac{1}{2} t_{R}^{(b)}=\frac{1}{8} t_{R}^{(c)}\right)$. The loss of the initial purity of the vibrational mode is reflected in Fig. 3(b) in which the time evolution of the entanglement parameter $S_{b}^{\text {corr }}$ is presented. From this figure we also see that a partial restoration of the initial quantum-statistical properties of the vibrational mode can be observed at the time $t_{R}^{(b)}$. Namely, in Fig. 4(b) we see that the one-peak structure of the $Q_{b}$ function is recovered at this moment (for $\gamma^{2}, \beta^{2} \gg 0$ ). This means that $t_{R}^{(b)}$ can be associated with the restoration (revival) time of the $Q_{b}$ function in phase space. Analogously $t_{R}^{(c)}$ is associated with the revival in phase space of the quantized cavity mode, i.e., with a partial restoration of the initial shape of the $Q_{c}$ function. In other words, the time scales $t_{R}^{(b)}$ and $t_{R}^{(c)}$ represent the main characteristics of the dynamics in those particular phase spaces that are not affected by quantum entanglement.

For completeness we mention that for small intensities of the cavity field mode $(\gamma \simeq 1)$ the $Q_{b}$ function of the vibrational mode splits into three rather than two components as in the case when $\gamma \gg \beta$ [compare Figs. 5(a) and 4(a), respectively]. One of the three peaks is "stationary" in the given rotating frame, while the other two peaks move clockwise and anticlockwise around the origin of the phase space. The existence of the stationary peak is associated with the presence of the state $|\beta\rangle_{b}|0\rangle_{c}|g\rangle_{i}$, which does not evolve under the action of the Hamiltonian (3.1). We note that analogous behavior in a three-level atomic system has already been seen by Knight and Shore [34].

One can also demonstrate that in the model (2) with a classical light field a considerable degree of squeezing of fluctuations of the position of the trapped ion can be found at the revival time $t_{R}^{(b)}$ [26]. In the model (3) with a quantized cavity field mode being initially coherently excited, this squeezing behavior is less pronounced, with moderate squeezing and sub-Poissonian statistics (amplitude fluctua- tions reduced below the coherent-state level) appearing at the initial stages of time evolution.

\section{B. Generalizations of the model}

In recent experiments with trapped ions [8] one can choose the type of the generalized Jaynes-Cummings-like interaction by tuning the laser field to an appropriate resolved vibrational sideband [19]. In particular, tuning a laser to the first lower (red) sideband means that in the LambDicke limit dynamics of the trapped ion interacting with a classical light field is described by the effective Hamiltonian $\hat{H}_{\text {c.l. }}^{\text {(red) }}=(i / 2) \hbar \Omega \epsilon\left[\hat{b}^{\dagger} \hat{\sigma}_{-}-\hat{b} \hat{\sigma}_{+}\right]$. When the cavity field is considered to be quantized, then the effective Hamiltonian reads $\hat{H}_{\mathrm{q} . \mathrm{f}}^{\text {(red }}=(i / 2) \hbar \kappa \epsilon\left[\hat{b}^{\dagger} \hat{c}^{\dagger} \hat{\sigma}_{-}-\hat{b} \hat{c} \hat{\sigma}_{+}\right]$. We note that if the system under consideration is initially prepared in a state $|\psi(0)\rangle=|0\rangle_{b} \otimes|0\rangle_{c} \otimes|e\rangle_{i}$ then at half of the generalized Rabi cycle it will evolve into the Greenberger-HorneZeilinger state [11] $\left|\psi\left(t_{h}\right)\right\rangle=(1 / \sqrt{2})\left(|0\rangle_{b}|0\rangle_{c}|e\rangle_{i}\right.$ $+|1\rangle_{b}|1\rangle_{c}|g\rangle_{i}$ ) at $\frac{1}{2} \kappa \epsilon t_{h}=\pi / 4$. This seems to us to be one of the most straightforward ways to realize a GHZ state in quantum optics.

When the laser field is tuned to the second upper vibrational sideband the effective Hamiltonian describing interaction of an ion with a classical light field in the Lamb-Dicke limit reads $\hat{H}_{\text {c.l. }}^{(2 n)}=\frac{1}{4} \hbar \Omega \epsilon^{2}\left[\hat{b}^{\dagger 2} \hat{\sigma}_{+}-\hat{b}^{2} \hat{\sigma}_{-}\right]$. This twophonon model is almost completely periodic (for a theoretical description of the two-photon JCM model see [35] and for a recent experimental realization of the ion-trap version of this model see the paper by Wineland and co-workers [9]). The periodicity is given by the specific dependence of the Rabi frequency on the vibration number $m$, i.e., the Rabi frequency is proportional to $\sqrt{m(m+1)}$, which implies that its values are commensurate for various values of $m \gg 1$. Therefore for $\beta^{2} \gg 1$ the time evolution is quasiperiodic. The revival time $t_{R}^{(2)}=4 \pi /\left(\Omega \epsilon^{2}\right)$ corresponds to the restoration of the initial $Q_{b}$ function (just rotated by $\pi$ ) and to the ion in the opposite internal state. One peculiarity of the two-photon model is that at one-half of the revival time, the vibrational mode is in a mixture state as $S_{b}^{\text {corr }} \approx 1 / 2$. On the other hand, the entanglement parameter is close to zero at $\frac{1}{4} t_{R}$ and $\frac{3}{4} t_{R}$, i.e., Schrödinger's catlike states with components mutually rotated by $\pi / 2$ are established. With the quantized cavity field, the effective two-phonon interaction is described by the Hamiltonian $\hat{H}_{\mathrm{q} . \mathrm{f} .}^{(2 \mathrm{nd})}=\frac{1}{4} \hbar \kappa \epsilon^{2}\left[\hat{b}^{\dagger 2} \hat{c} \hat{\sigma}_{-}-\hat{b}^{2} \hat{c}^{\dagger} \hat{\sigma}_{+}\right]$. We briefly note that this nonlinear interaction between three quantum subsystems results in very complex dynamics. In particular, let us assume that the cavity field mode is initially prepared in a coherent state $|\gamma\rangle_{c}$. In this case the parameter $S_{i}^{\text {corr }}$ describing the entanglement between the internal degree of freedom of the ion and the two bosonic modes (i.e., the vibrational mode and the cavity field) converges to the stationary value of $1 / 2$ via a sequence of minima at times $[(2 n+1) 4] t_{R}^{(2)}$, where $t_{R}^{(2)}=4 \pi /\left(\kappa \gamma \epsilon^{2}\right)$. At the first minimum $\frac{1}{4} t_{R}^{(2)}$ the value of $S_{i}^{\text {corr }}$ is close to zero (for $\beta^{2}, \gamma^{2} \gg 1$ ), which means a coherent superposition of the two internal ionic levels is created at this moment. Simultaneously, the combined system of the vibrational mode and the cavity field is in a pure state - the two-mode Schrödinger catlike state (see for instance [36]). 


\section{TWO TRAPPED TWO-LEVEL IONS IN A CAVITY}

In the previous section we have analyzed dynamics of the trapped two-level ion interacting with the quantized cavity field. Now we address the question of what will happen when a cluster of trapped two-level ions interacts with the same cavity mode. Recent work on collections of more than one trapped ion has already demonstrated the existence of observable collective effects (see [37] and references therein).

For this purpose we generalize the Hamiltonian (3) and we propose the interaction Hamiltonian for a cluster of trapped two-level ions interacting with a quantized cavity mode:

$$
\hat{H}_{\mathrm{q} . \mathrm{f} .}^{(N)}=\frac{i}{2} \hbar \epsilon \sum_{j=0}^{N} \kappa_{j}\left[\hat{b}_{j}^{\dagger} \hat{c} \hat{\sigma}_{+}^{(j)}-\hat{b}_{j} \hat{c}^{\dagger} \hat{\sigma}_{-}^{(j)}\right]
$$

This Hamiltonian is written in the rotating frame associated with the light field and it describes the dynamics of trapped ions in the Lamb-Dicke limit. In Eq. (4.1) $\hat{b}_{j}\left(\hat{b}_{j}^{\dagger}\right)$ denotes the annihilation (creation) operator of the vibrational motion of the $j$ th ion described by the spin-flip operators $\hat{\sigma}_{ \pm}^{(j)}$. We focus our attention on comparison of the results for the model describing one trapped ion $(N=1)$ and the results in the case of two identical ions $(N=2)$. To simplify our discussion we assume that the ions interact with the cavity field with the same intensity, i.e., $\kappa=\kappa_{1}=\kappa_{2}$. This model could address the situation when two ion traps (each with a single ion) are enclosed by the same resonator; i.e., they share a quantized cavity field mode that mediates an indirect coupling between the ions. We could extend our analysis to describe laser-cooled atoms trapped in optical lattices [38]. The optical potential generated for neutral atoms in standingwave laser beams possesses sufficient periodic structure for the effects we have been concerned with to be relevant and thus one can even imagine the case when the atoms are localized at the sites of different potential wells.

We start our analysis with an observation that if the cavity field is initially prepared in a coherent state and the ions are in their lower internal states and, in addition, if we assume the ions to be in the Fock (number) vibrational states, i.e.,

$$
|\psi(0)\rangle=|\gamma\rangle_{c} \otimes\left|M_{1}\right\rangle_{b}^{(1)}|g\rangle_{i}^{(1)} \otimes\left|M_{2}\right\rangle_{b}^{(2)}|g\rangle_{i}^{(2)}
$$

then the dynamics of the cavity field is the same as that within the framework of the collective Tavis-Cummings model [39], with the interaction Hamiltonian

$$
\hat{H}_{\mathrm{TCM}}^{(N)}=\hbar \sum_{j=1}^{N}\left[\lambda \hat{c} \hat{\sigma}_{+}^{(j)}+\lambda * \hat{c}^{\dagger} \hat{\sigma}_{-}^{(j)}\right] .
$$

This isomorphism between the two models is valid providing the interaction constant $\lambda$ is given by the relation $\lambda=(i / 2) \kappa \epsilon \sqrt{\left(M_{1}+1\right)\left(M_{2}+1\right)}$. In this case we observe an interesting effect: splitting of the $Q_{c}$ function of the cavity field into three components (or, generally, into $N+1$ components if the cluster consists of $N$ ions) [40]. The time at which the $Q_{c}$ function of the cavity mode returns again to its initial shape is equal to the time at which the revival of the mean photon number appears and is given by the relation
$t_{R}=2 \pi \gamma /|\lambda|$. The time evolution of populations of the internal ionic levels is also the same as within the TavisCummings model but any kind of ionic coherence between collective Dicke levels [41] is inevitably lost owing to the perfect entanglement of the internal ionic energy states with the vibrational number states. Namely, the states $\left.\left.\| g(N)\rangle\rangle^{(j)}=\left|M_{j}\right\rangle_{b}^{(j)}|g\rangle_{i}^{(j)}, \quad \| e(N)\right\rangle\right\rangle^{(j)}=\left|M_{j}+1\right\rangle_{b}^{(j)}|e\rangle_{i}^{(j)}$ $(j=1,2)$ form collective ionic states. Consequently, the vibrational mode of each ion is for $t>0$ in a statistical mixture.

Further we assume that all bosonic modes of the model are initially in coherent states, i.e.,

$$
|\psi(0)\rangle=|\gamma\rangle_{c} \otimes\left|\beta_{1}\right\rangle_{b}^{(1)}|g\rangle_{i}^{(1)} \otimes\left|\beta_{2}\right\rangle_{b}^{(2)}|g\rangle_{i}^{(2)} .
$$

Because the model [given by Eq. (4.1)] totally neglects mutual ionic collisions, it is natural to assume that initially the ions are "localized" at different sites within their respective traps. When one of the trapped ions is cooled down to the zero-point vibrational energy, e.g., $\beta_{1}=\beta$ and $\beta_{2}=0$, then the vibrational motion of the first ion is not affected by the presence of the second ion, which does not then enter the interaction governed by Eq. (4.1). We have studied the nontrivial case $\beta_{1}=-\beta_{2}=\beta$, when the ions are initially "localized" at opposite sides of their respective traps. We remind ourselves that the initial average number of vibrational quanta $\bar{n}_{b}=\beta^{2}$ in each of the vibrational modes has to be chosen to obey the condition $\epsilon^{2} \bar{n}_{b} \ll 1$ to ensure that we operate in the Lamb-Dicke limit. The results obtained for $\gamma \gg 1$ suggest that vibrational motion of one ion is not affected by the presence of other ions. The characteristic (revival) time $t_{R}^{(b)}$ in the vibrational phase space of each ion is again equal to $t_{R}^{(b)} \approx 4 \pi \beta /(\kappa \epsilon \gamma)$ [see Eq. (3.13)]. In other words, during a time period of the order of few times $t_{R}^{(b)}$ the ions evolve independently. Only the maximum value of the entanglement parameter of the cavity field increases due to the direct coupling of the cavity mode to more subsystems.

It is interesting to note that if $\beta_{1}=\beta_{2}=\beta$ (e.g., the two ions are initially located at equivalent points of two spatially separated traps which are coupled through the cavity field of the resonator) the $Q_{b}$ function in the corresponding vibrational phase space has the same dynamics (except a phase shift) as in the case when initially $\beta_{1}=-\beta_{2}=\beta$.

For completeness we should comment in some detail on the behavior of the cavity field. The time evolution of the cavity field depends on the initial average numbers $\bar{n}_{b}^{(1)}$ and $\bar{n}_{b}^{(2)}$ of vibrational quanta. If the time scales $t_{R}^{(1)}$ $=4 \pi\left[\bar{n}_{b}^{(1)}\right]^{1 / 2} /(\kappa \epsilon \gamma)$ and $t_{R}^{(2)}=4 \pi\left[\bar{n}_{b}^{(2)}\right]^{1 / 2} /(\kappa \epsilon \gamma)$ are of the same order then the $Q_{c}$ function of the field mode splits into three components with one dominant static peak. Otherwise there are two independent bifurcation processes that result in a splitting of the $Q_{c}$ function into four components. This reflects the fact that the cavity mode interacts with each ion independently.

\section{CONCLUSIONS}

One of the main results of our investigation is that in the nonclassical Lamb-Dicke limit the time evolution of the vibrational mode representing the quantized motion of the center of mass of the trapped ion is very sensitive to the nature 
of the light field. If the light field is treated quantum mechanically, then a strong entanglement between the ionic internal and vibrational degrees of freedom and the light field leads to a change in the quantum-statistical properties of the vibrational mode. Nevertheless, an isomorphism between the two models described by the Hamiltonians (2) and (3) is achieved when the quantized light field is initially in a Fock state with a precisely defined number of photons. In this casea pure superposition state of the vibrational mode is produced at one-half of the characteristic time $t_{R}$. The other subsystems (i.e., the cavity mode and the internal degree of freedom of the ion) are in statistical mixtures, which is in contrast with the behavior of the system described by the model (2) with the classical light field.

When the quantized cavity mode is initially in other than a Fock state then the initial purity of the vibrational mode is inevitably lost due to the entanglement between the quantum-mechanical subsystems. Consequently, instead of the superposition state a statistical mixture in the vibrational mode is created. Also the cavity field evolves into a statistical mixture. On the other hand, owing to the entanglement a two-mode superposition state in the system composed of vibrational and cavity field modes can be established in certain circumstances. We found that two different time scales characterize dynamics of the quantum-mechanical system. Namely, as soon as the cavity mode is initially prepared in a superposition of Fock states $|n\rangle_{c}$, i.e., $|\psi(0)\rangle_{c}=\Sigma c_{n}|n\rangle_{c}$, then the revival times $t_{R}^{(b)}(n)=4 \pi \beta /(\kappa \epsilon \sqrt{n})$ [see Eq. (3.10)] for different values of $n$ do not match and the amplitudes of corresponding revivals (at $t_{R}^{(b)}$ and its multiples) of the average number of trap quanta become modulated on the time scale associated with $t_{R}^{(c)}$. As a result in the limit of high intensity of the quantum light field (i.e., $\gamma \rightarrow \infty$ ) all revivals are smeared out. On the contrary, if the light field is treated classically, then in the high-intensity limit the revivals of the vibrational mode are perfectly preserved.

From our results it follows that the increase in the number of trapped ions that interact with the quantized cavity mode within the model (4.1) does not influence the dynamics of the ions on the time scale $t_{R}^{(b)}$ providing the number of ions is much smaller than the mean photon number of the cavity field mode and the mean number of trap quanta. We have pointed out further modifications of the model with a quantized cavity field mode. They can be of interest in the creation of fully correlated Greenberger-Horne-Zeilinger states as well as for transfer of information between trapped ions though a quantum channel provided by a quantized cavity field mode.

Finally, the physical situation described by the model Hamiltonian (3.1) requires a combination of the strong coupling regime of cavity QED with the resolved sideband excitation of a trapped ion. Whereas strong coupling is realized typically with a strong (dipole) transition, resolved sideband excitation is performed on a weak (quadrupole or Raman) transition. In order to realize a transition with both strong coupling and resolved sidebands one would need to ensure a radio frequency drive to the trap high enough such that secular frequencies are sufficiently large. There is no reason in principle why such a trap cannot be built [42]. For instance, high-frequency combined rf Penning traps have been recently reported by Hänsch and co-workers [43]. Such problems of realization take us beyond the scope of the present paper, which is concerned with conceptual matters.

\section{ACKNOWLEDGMENTS}

We thank R. Blatt, D. Segal, R. Thompson, H. Walther, and $\mathrm{P}$. Zoller for useful discussions and correspondence. This work was supported by the United Kingdom Engineering and Physical Sciences Research Council, the European Community, by the East-West Program of the Austrian Academy of Sciences under Contract No. 45.367/6-IV/3a/95 of the Osterreichisches Bundesministerium für Wissenschaft und Forschung, and by the Grant Agency VEGA of the Slovak Academy of Sciences under the Grant 2/1152/96. M.S.K. is grateful to the Alexander von Humboldt Foundation for support.
[1] S. Haroche, in Fundamental Systems in Quantum Optics, Les Houches session LIII, edited by J. Dalibard, J.-H. Raimond, and J. Zinn-Justin (North-Holland, Amsterdam, 1992), p. 767.

[2] G. Rempe, R. J. Thompson, and H. J. Kimble, Phys. Scr. T51, 67 (1994); R. J. Brecha, L. A. Orozco, M. Xiao, and H. J. Kimble, J. Opt. Soc. Am. B 12, 2319 (1995); see also a review article G. Rempe, Contemp. Phys. 34, 119 (1993).

[3] J. Gripp, S. L. Mielke, L. A. Orozco, and H. J. Carmichael, Phys. Rev. A 54, R3746 (1996).

[4] Priviate communications with R. Blatt (Innsbruck), D. Segal and R. Thompson (London), and H. Walther (Munchen); see also G. M. Meyer, H.-J. Briegel, and H. Walther, Europhys. Lett. 37, 317 (1997).

[5] For overview see, e.g., lectures by C. Cohen-Tannoudji and W. D. Phillips on laser cooling of neutral atoms and lectures by $\mathrm{H}$. Walther and R. Blatt on laser cooling of trapped ions, in Fundamental Systems in Quantum Optics, 1990 Les Houches Session No. LIII, edited by J. Dalibard, J.-M. Raimond, and J.
Zinn-Justin (North-Holland, Amsterdam, 1992).

[6] F. Diedrich, J. C. Bergquist, W. M. Itano, and D. J. Wineland, Phys. Rev. Lett. 62, 403 (1989).

[7] C. Monroe, D. M. Meekhof, B. E. King, S. R. Jeffers, W. M. Itano, D. J. Wineland, and P. Gould, Phys. Rev. Lett. 75, 4011 (1995).

[8] D. M. Meekhof, C. Monroe, B. E. King, W. M. Itano, and D. J. Wineland, Phys. Rev. Lett. 76, 1796 (1996).

[9] D. Leibfried, D. M. Meekhof, C. Monroe, B. E. King, W. M. Itano, and D. J. Wineland, J. Mod. Opt. (to be published).

[10] G. Drobný, V. Bužek, and M.S. Kim, in Coherence and Quantum Optics VII, edited by L. Mandel, E. Wolf, and J. H. Eberly (Plenum, New York, 1996), p. 381.

[11] D. M. Greenberger, M. H. Horne, and A. Zeilinger, Bell's Theorem, Quantum Theory, and Conceptions of the Universe, edited by M. Kafatos (Kluwer, Dordrecht, 1989); Phys. Today 46(8), 22 (1993).

[12] C. A. Blockley, D. F. Walls, and H. Risken, Europhys. Lett. 17, 509 (1992). 
[13] J. I. Cirac, R. Blatt, P. Zoller, and W. D. Phillips, Phys. Rev. A 46, 2668 (1992); J. I. Cirac, R. Blatt, A. S. Parkins, and P. Zoller, ibid. 48, 2169 (1993); Phys. Rev. Lett. 70, 762 (1993); J. I. Cirac, A. S. Parkins, R. Blatt, and P. Zoller, ibid. 70, 556 (1993).

[14] E. T. Jaynes and F. W. Cummings, Proc. IEEE 51, 89 (1963); for a review see, e.g., B. Shore, and P. L. Knight, J. Mod. Opt. 40, 1195 (1993).

[15] W. Vogel, and R. L. de Matos Filho, Phys. Rev. A 52, 4214 (1995); R. L. de Matos Filho, and W. Vogel, ibid. 50, R1988 (1994); Phys. Rev. Lett. 76, 608 (1996).

[16] H. Zeng and F. Lin, Phys. Rev. A 50, R3589 (1994); On transfer of quantum information between two vibrational modes of a trapped ion, see J. Steinbach, J. Twamley, and P. L. Knight (unpublished).

[17] R. L. de Matos Filho and W. Vogel, Phys. Rev. Lett. 76, 4520 (1996).

[18] S. Wallentowitz and W. Vogel, Phys. Rev. Lett. 75, 2932 (1995); J. F. Poyatos, R. Walser, J. I. Cirac, and P. Zoller, Phys. Rev. A 53, R1966 (1996); C. D’Helon and G. J. Milburn, ibid. 53, R25 (1996); P. J. Bardroff, C. Leichtle, G. Schrade and W. P. Schleich, Phys. Rev. Lett. 77, 2198 (1996).

[19] D. Leibfried, D. M. Meekhof, B. E. King, C. Monroe, W. M. Itano, and D. J. Wineland, Phys. Rev. Lett. 77, 4281 (1996); C. Monroe, D. M. Meekhof, B. E. King, D. Liebfried, W. M. Itano, and D. J. Wineland, Acc. Chem. Res. 29, 585 (1996).

[20] T. Pellizzari, S. A. Gardiner, J. I. Cirac, and P. Zoller, Phys. Rev. Lett. 75, 3788 (1995).

[21] Q. A. Turchette, C. J. Hood, W. Lange, H. Mabuchi, and H. J. Kimble, Phys. Rev. Lett. 75, 4710 (1996).

[22] C. K. Law and J. H. Eberly, Phys. Rev. Lett. 76, 1055 (1996).

[23] P. J. Bardroff, E. Mayr, and W. P. Schleich, Phys. Rev. A 51, 4963 (1995); P. J. Bardroff, E. Mayr, W. P. Schleich, P. Domokos, M. Brune, J. M. Raimond, and S. Haroche, Phys. Rev. A 53, 2736 (1996).

[24] J. I. Cirac and P. Zoller, Phys. Rev. Lett. 74, 4091 (1995).
[25] J. I. Cirac, L. J. Garay, R. Blatt, A. S. Parkins, and P. Zoller, Phys. Rev. A 49, 421 (1994).

[26] H. Zeng and F. Lin, Phys. Rev. A 48, 2393 (1993).

[27] S. J. D. Phoenix and P. L. Knight, Ann. Phys. (N.Y.) 186, 381 (1988); V. Bužek, H. Moya-Cessa, P. L. Knight, and S. J. D. Phoenix, Phys. Rev. A 45, 8190 (1992).

[28] J. Gea-Banacloche, Phys. Rev. Lett. 65, 3385 (1990); Phys. Rev. A 44, 5913 (1991).

[29] V. Bužek and P. L. Knight, in Progress in Optics XXXIV, edited by E. Wolf (North-Holland, Amsterdam, 1995), p. 1, and references therein.

[30] S.-C. Gou and P. L. Knight, Phys. Rev. A 54, 1682 (1996); S.-C. Gou, J. Steinbach, and P. L. Knight, ibid. 54, 4315 (1996).

[31] C. C. Gerry and J. H. Eberly, ibid. 42, 6805 (1990).

[32] A. Wehrl, Rev. Mod. Phys. 50, 221 (1978).

[33] K. E. Cahill and R. J. Glauber, Phys. Rev. 177, 1857 (1969); 177, 1882 (1969).

[34] P. L. Knight and B. W. Shore, Phys. Rev. A 48, 642 (1993).

[35] C. C. Gerry, Phys. Rev. A 37, 2683 (1988); C. C. Gerry and P. J. Moyer, ibid. 38, 5665 (1988).

[36] C. C. Gerry and R. Grobe, J. Mod. Opt. 44, 41 (1997).

[37] R. G. Brewer, Phys. Rev. Lett. 77, 5153 (1996).

[38] A. Hemmerich and T. W. Hänsch, Phys. Rev. Lett. 70, 410 (1993); A. Hemmerich, M. Weidemuller, T. Esslinger, C. Zimmermann, and T. W. Hänsch, ibid. 75, 37 (1995); T. Esslinger, F. Sander, A. Hemmerich, T. W. Hänsch, H. Ritsch, and M. Weidemuller, Opt. Lett. 21, 991 (1996).

[39] M. Tavis and F. W. Cummings, Phys. Rev. 170, 379 (1968); 188, 692 (1969).

[40] G. Drobný and I. Jex, Opt. Commun. 102, 141 (1993); G. Drobný, Ts. Gantsog, and I. Jex, Phys. Rev. A 48, 622 (1994).

[41] R. Dicke Phys. Rev. A 93, 99 (1954).

[42] D. Segal (private communication).

[43] J. Walz, S. B. Ross, C. Zimmermann, L. Ricci, M. Prevedelli, and T. W. Hänsch, Phys. Rev. Lett. 75, 3257 (1995); J. Walz, S. B. Ross, C. Zimmermann, L. Ricci, M. Prevedelli, and T. W. Hänsch, Hyperfine Interact. 100, 133 (1996). 